\title{
Strong conciseness of coprime and anti-coprime commutators
}

\author{
Eloisa Detomi, Marta Morigi, and Pavel Shumyatsky
}

\begin{abstract}
A coprime commutator in a profinite group $G$ is an element of the form $[x, y]$, where $x$ and $y$ have coprime order and an anti-coprime commutator is a commutator $[x, y]$ such that the orders of $x$ and $y$ are divisible by the same primes. In the present paper we establish that a profinite group $G$ is finite-by-pronilpotent if the cardinality of the set of coprime commutators in $G$ is less than $2^{\aleph_{0}}$. Moreover, a profinite group $G$ has finite commutator subgroup $G^{\prime}$ if the cardinality of the set of anti-coprime commutators in $G$ is less than $2^{\aleph_{0}}$.
\end{abstract}

\section{Introduction}

Let $w=w\left(x_{1}, \ldots, x_{k}\right)$ be a group-word, that is, a nontrivial element of the free group on $x_{1}, \ldots, x_{k}$. The word $w$ can be naturally viewed as a function of $k$ variables defined on any group $G$. The subgroup generated by the set of $w$-values is called the verbal subgroup corresponding to the word $w$. We denote this by $w(G)$. In the context of topological groups $G$ we write $w(G)$ to denote the closed subgroup generated by all $w$-values in $G$.

The word $w$ is said to be concise in the class of groups $\mathcal{C}$ if the verbal subgroup $w(G)$ is finite whenever $w$ takes only finitely many values in a group $G \in \mathcal{C}$ (see for example [4, [5] and references therein for results on conciseness of words). A natural variation of this notion for profinite groups was recently introduced in [3]: we say that $w$ is strongly concise in a class of profinite groups $\mathcal{C}$ if the verbal subgroup $w(G)$ is finite in any group $G \in \mathcal{C}$ in which $w$ takes less than $2^{\aleph_{0}}$ values.

2020 Mathematics Subject Classification. 20E18; 20D25.

Key words and phrases. Commutators, conciseness, profinite groups, pronilpotent groups. 
A number of new results on strong conciseness of group-words can be found in 3 .

The concept of (strong) conciseness can be applied in a much wider context. Suppose $\mathcal{C}$ is a class of profinite groups and $\varphi(G)$ is a subset of $G$ for every $G \in \mathcal{C}$. One can ask whether the subgroup generated by $\varphi(G)$ is finite whenever $|\varphi(G)|<2^{\aleph_{0}}$.

In the present paper we establish strong conciseness of the sets of coprime and anti-coprime commutators. By a coprime commutator in a profinite group $G$ we mean any element of the form $[x, y]$, where $\pi(x) \cap \pi(y)=\emptyset$, and by an anti-coprime commutator we mean any commutator $[x, y]$ such that $\pi(x)=\pi(y)$. Here and throughout the paper $\pi(x)$ stands for the set of prime divisors of the order of the procyclic group generated by $x$.

It is well-known that in any profinite group $G$ the coprime commutators generate the pronilpotent residual $\gamma_{\infty}(G)$, that is, the (unique) minimal normal subgroup $N$ such that $G / N$ is pronilpotent. So, if a profinite group is finite-by-pronilpotent, then it has only finitely many coprime commutators. Our main result is that also the converse holds.

THEOREM 1.1. A profinite group $G$ is finite-by-pronilpotent if and only if the cardinality of the set of coprime commutators in $G$ is less than $2^{\aleph_{0}}$.

Remark that in the particular case of the group $G$ with only finitely many coprime commutators the above theorem is straightforward from the main result of $[\mathbf{1}$.

The set of anti-coprime commutators generates the commutator subgroup $G^{\prime}$ of $G$. Hence if $G^{\prime}$ is finite, then so is this set. As a consequence of the results in [3], we have the following new characterization of finite-by-abelian groups.

TheOREM 1.2. A profinite group $G$ has finite commutator subgroup $G^{\prime}$ if and only if the cardinality of the set of anti-coprime commutators in $G$ is less than $2^{\aleph_{0}}$.

It follows from the proof of Theorem 1.2 that if the profinite group $G$ has only finitely many, say $m$, anti-coprime commutators, then the order of $G^{\prime}$ is $m$-bounded. Throughout the paper we use the expression " $m$-bounded" to mean that a quantity is finite and bounded by a certain number depending only on the parameter $m$.

To conclude this short introduction, we mention a related problem. Let $\gamma_{k}(G)$ denote the $k$ th term of the lower central series of a group $G$ and $G^{(k)}$ that of the derived series of $G$. In view of our results it would 
be interesting to find a small strongly concise set of generators for each of these subgroups.

\section{Preliminaries}

Many results of the theory of finite groups admit a natural interpretation for profinite groups. Throughout the paper we use certain profinite versions of facts on finite groups without explaining in detail how the results on profinite groups can be deduced from the corresponding finite cases. On all such occasions the deduction can be performed via the routine inverse limit argument.

Our notation is standard (see, for example, [11] or [14]). Every homomorphism of profinite groups considered in this paper is continuous, and every subgroup of a profinite group is closed.

If $G$ is a profinite group, then $|G|$ denotes its order, which is a Steinitz number, and $\pi(G)$ denotes the set of prime divisors of $|G|$. Similarly, if $g$ is an element of $G$, then $|g|$ and $\pi(g)$ respectively denote the order of the procyclic subgroup generated by $g$ and the set of prime divisors of $|g|$.

Profinite groups have $p$-Sylow subgroups and satisfy analogues of the Sylow theorems. Prosoluble groups have $\pi$-Hall subgroups and Sylow bases. If $G$ is a profinite group and $\pi$ a set of primes, $O_{\pi}(G)$ stands for the maximal normal pro- $\pi$ subgroup of $G$.

Each profinite group $G$ has a maximal pronilpotent normal subgroup, its Fitting subgroup $F(G)$. Set $F_{0}(G)=1$ and $F_{i+1}(G) / F_{i}(G)=$ $F\left(G / F_{i}(G)\right)$ for every integer $i \geq 0$. A profinite group $G$ is metapronilpotent if and only if it $F_{2}(G)=1$ or, equivalently, if and only if $\gamma_{\infty}(G)$ is pronilpotent.

One can easily see that if $N$ is a normal subgroup of $G$ and $g$ is an element whose image in $G / N$ is a coprime (resp. anti-coprime) commutator, then there exists a coprime (resp. anti-coprime) commutator $h$ such that $g \in h N$. It follows that if a profinite group has less than $2^{\aleph_{0}}$ coprime (resp. anti-coprime) commutators, then this condition is inherited by every homomorphic image of the group, and we shall use this property without special reference.

Lemma 2.1. 3, Lemma 2.2] Let $G$ be a profinite group and let $x \in G$. If the conjugacy class $x^{G}$ contains less than $2^{\aleph_{0}}$ elements, then it is finite.

The following lemma is an important observation used throughout this paper. 
LEMMA 2.2. Let $G$ be a profinite group in which the set of coprime commutators contains less than $2^{\aleph_{0}}$ elements. If $x \in G$ and $H$ is a subgroup of $G$ such that $(|x|,|H|)=1$, then $C_{H}(x)$ has finite index in $H$.

Proof. Since $[x, h]=x^{-1} x^{h}$, the cardinality of the set $x^{H}=\left\{x^{h} \mid\right.$ $h \in H\}$ is equal to the cardinality of the subset $\{[x, h] \mid h \in H\}$ of the set of coprime commutators of $G$. Thus $x^{H}$ contains less than $2^{\aleph_{0}}$ elements. As $x^{H}$ is in bijection with the coset space $H / C_{H}(x)$, a homogeneous profinite space, we conclude that $x^{H}$ is finite.

If a group $A$ acts on the group $G$ via automorphisms, the subgroup generated by elements of the form $g^{-1} g^{a}$ with $g \in G$ and $a \in A$ is denoted by $[G, A]$. It is well-known that the subgroup $[G, A]$ is an $A$-invariant normal subgroup of $G$. The symbol $\Phi(G)$ stands for the Frattini subgroup of a group $G$.

The following result is well-known and will be often used without reference.

Lemma 2.3. 6. Lemma 4.29] Let $A$ act via automorphisms on $G$, where $A$ and $G$ are finite groups such that $(|G|,|A|)=1$. Then $[G, A, A]=[G, A]$.

LEMMA 2.4. Let $G=Q H$ be a group, where $H$ is a subgroup and $Q$ is a normal subgroup of $G$ such that $Q=[Q, H]$. If $N$ is a normal subgroup of $Q$ such that $[N, H]=1$, then $N$ is contained in the center of $G$.

Proof. By the Three Subgroup Lemma (see [12, 5.1.10]) we have that $[Q, H, N] \leq[H, N, Q][N, Q, H] \leq[H, N]=1$. Thus, $N$ centralizes $[Q, H]=Q$ and the result follows.

Lemma 2.5. [8, Lemma 2.3] Let $V$ be an elementary abelian finite p-group, and $U$ a $p^{\prime}$-group of automorphisms of $V$. If $|[V, u]| \leq m$ for every $u \in U$, then $|[V, U]|$ is m-bounded, and therefore $|U|$ is also m-bounded.

Lemma 2.6. Assume that $G=Q H$ is a finite group with a normal nilpotent subgroup $Q$ and a subgroup $H$ such that $(|Q|,|H|)=1$ and $\left|Q: C_{Q}(x)\right| \leq m$ for all $x \in H$. Then the order of $[Q, H]$ is $m$-bounded.

Proof. As $[Q, H, H]=[Q, H]$ by Lemma 2.3 , we can replace $Q$ with $[Q, H]$ and simply assume that $Q=[Q, H]$. Consequently, as $Q$ is the direct product of its Sylow subgroups, $P=[P, H]$ for each Sylow subgroup $P$ of $Q$. As $\left|P: C_{P}(x)\right| \leq\left|Q: C_{Q}(x)\right| \leq m$, it follows that $H$ acts trivially on $P$ whenever $p>m$. Taking into account that 
$P=[P, H]$ we conclude that all prime divisors of the order of $Q$ are at most $m$. Hence, the number of Sylow subgroups of $Q$ is $m$-bounded. Therefore, without loss of generality, we may assume that $Q$ is a $p$ group and $H$ acts faithfully on $Q$. As $(|Q|,|H|)=1$, by $[\mathbf{2}$, (24.1)] the action on $Q / \Phi(Q)$ is faithful. So, by Lemma 2.5 we obtain that $|H|$ is $m$-bounded. It follows that $\left|Q: C_{Q}(H)\right|$ is $m$-bounded. Let $N$ be the intersection of all conjugates in $G$ of $C_{Q}(H)$. Then $N$ is a normal subgroup of $G$ of $m$-bounded index. We deduce from Lemma 2.4 that $N$ is a subgroup of the center of $G$. Thus, Schur's theorem [13, 4.12] tells us that $G^{\prime}$ has finite $m$-bounded order, and the lemma follows.

\section{Coprime commutators}

In this section we analize the structure of profinite groups with less than $2^{\aleph_{0}}$ coprime commutators. We wish to prove that these commutators generate a finite subgroup.

LemMA 3.1. Let $G$ be a profinite group with less than $2^{\aleph_{0}}$ coprime commutators. Assume that $G$ is a product of a subgroup $H$ and a normal pronilpotent subgroup $Q$ with $(|Q|,|H|)=1$. Then $[Q, H]$ is finite.

Proof. By the profinite version of Lemma 2.3 we have $[Q, H, H]=$ $[Q, H]$. So we can replace $Q$ with $[Q, H]$ and assume that $Q=[Q, H]$. By Lemma 2.2, $\left|Q: C_{Q}(x)\right|$ is finite for any $x \in H$. For each positive integer $j$ consider the set:

$$
C_{j}=\left\{x \in H|| x^{Q} \mid \leq j\right\} .
$$

Note that the sets $C_{j}$ are closed (see for instance [9, Lemma 5]). As the union of the sets $C_{j}$ is $H$, by the Baire category theorem (cf. [7, p.200]) at least one of the sets $C_{j}$ has non-empty interior with respect to $H$. So there exists a positive integer $s$, an open normal subgroup $J$ of $H$ and an element $a \in H$ such that $\left|(a x)^{Q}\right| \leq s$ for every $x \in J$. For $x \in J$ and $y \in Q$ write $x^{y}=\left(a^{-1}\right)^{y}(a x)^{y}$. Note that $\left|\left(a^{-1}\right)^{Q}\right| \leq s$ and $\left|(a x)^{Q}\right| \leq s$. Therefore $\left|Q: C_{Q}(x)\right| \leq s^{2}$ for any $x \in J$. By Lemma 2.6 we conclude that $[Q, J]$ is finite.

Replacing $Q$ with the quotient group $Q /[Q, J]$ and $H$ with the quotient group $H / J$, we can assume that $H$ is finite and thus also $\mid Q$ : $C_{Q}(H) \mid$ is finite. Note that $C_{Q}(H)$ has only finitely many conjugates in $G$. Let $N$ be the intersection of all conjugates of $C_{Q}(H)$. Clearly, $|Q: N|$ is finite. It follows from Lemma 2.4 that $N$ is a subgroup of the center of $G$. Thus the center of $G$ has finite index in $G$, and the result now follows from the theorem of Schur [13, 4.12]. 
LEMma 3.2. [1, Lemma 2.4] If $G$ is a finite metanilpotent group, then $\gamma_{\infty}(G)=\prod_{p}\left[K_{p}, H_{p^{\prime}}\right]$, where $K_{p}$ is a $p$-Sylow subgroup of $\gamma_{\infty}(G)$ and $H_{p^{\prime}}$ is a $p^{\prime}$-Hall subgroup of $G$.

LEMMA 3.3. Let $G$ be a metapronilpotent group with less than $2^{\aleph_{0}}$ coprime commutators. Then for each $p \in \pi\left(\gamma_{\infty}(G)\right)$ the $p$-Sylow subgroup of $\gamma_{\infty}(G)$ is finite.

Proof. Note that $\gamma_{\infty}(G)$ is pronilpotent. Choose $p \in \pi\left(\gamma_{\infty}(G)\right)$ and let $P$ be the $p$-Sylow subgroup of $\gamma_{\infty}(G)$. The profinite version of Lemma 3.2 implies that $P=[P, H]$, where $H$ is a $p^{\prime}$-Hall subgroup of $G$. Lemma 3.1 now shows that $P$ is finite, as required.

Recall that any prosoluble group $G$ has a Sylow basis (a family of pairwise permutable $p_{i}$-Sylow subgroups $P_{i}$ of $G$, exactly one for each prime $p_{i} \in \pi(G)$ ), and any two Sylow bases are conjugate (see [11. Proposition 2.3.9]). The basis normalizer (also known as the system normalizer) of such a Sylow basis in $G$ is $T=\cap_{i} N_{G}\left(P_{i}\right)$. If $G$ is a prosoluble group and $T$ is a basis normalizer in $G$, then $T$ is pronilpotent and $G=\gamma_{\infty}(G) T$ (see [10, Lemma 5.6]).

LEMma 3.4. Let $G$ be a metapronilpotent profinite group with less than $2^{\aleph_{0}}$ coprime commutators. Then $\gamma_{\infty}(G)$ is finite.

Proof. Let $K=\gamma_{\infty}(G)$. In view of Lemma 3.3 it is sufficient to show that $\pi(K)$ is finite.

Assume by contradiction that $\pi(K)$ is infinite. Let $T$ be a basis normalizer of a Sylow basis in $G$. Note that both $T$ and $K$ are pronilpotent.

Let $\pi(K)=\left\{p_{1}, p_{2}, \ldots, p_{i}, \ldots\right\}$ and for each $i=1,2, \ldots$ let $P_{i}$ be the $p_{i}$-Sylow subgroup of $K$. By Lemma 3.3 we know that the subgroups $P_{i}$ are finite. For each index $i$ observe that the group $O_{p_{i}^{\prime}}(T)$ naturally acts on $P_{i}$. Let $A_{i}$ be the group of automorphisms of $P_{i}$ induced by the action of $O_{p_{i}^{\prime}}(T)$. It follows from Lemma 3.2 that $P_{i}=$ $\left[P_{i}, A_{i}\right]$. Set $\sigma_{i}=\pi\left(A_{i}\right)$. Then $\sigma_{i}$ is a finite nonempty set. Let $q \in \pi(T)$ and let $T_{q}$ be a $q$-Sylow subgroup of $T$. By Lemma 3.1, the subgroup $\left[O_{q^{\prime}}(K), T_{q}\right]$ is finite. Therefore $q \in \sigma_{j}$ for only finitely many indices $j$. We denote the set $\left\{p_{i}\right\} \cup \sigma_{i}$ by $\tau_{i}$. So for every index $i$ there exists only finitely many indexes $j$ such that $\tau_{j} \cap \tau_{i} \neq \emptyset$.

Since $\pi(K)$ is infinite, we can choose an infinite set of indices $J$ such that $\tau_{i} \cap \tau_{j}=\emptyset$ whenever $i, j$ are different indices in $J$. For each $i \in J$ choose $q \in \sigma_{i}$ and let $Q_{i}$ be the $q$-Sylow subgroup of $T$. It follows that $\left[P_{i}, Q_{i}\right] \neq 1$ while $\left[P_{j}, Q_{i}\right]=1$ whenever $i, j \in J$ are such that $i \neq j$.

For each $i \in J$ choose $a_{i} \in P_{i}$ and $b_{i} \in Q_{i}$ such that $1 \neq\left[a_{i}, b_{i}\right]=c_{i}$. Obviously the element $c_{i}$ is a coprime commutator. Observe that by 
construction $\left\langle a_{i}, b_{i}\right\rangle$ commutes with $\left\langle a_{j}, b_{j}\right\rangle$ whenever $i \neq j$. Therefore for any subset $I \subseteq J$ the product $\prod_{i \in I} c_{i}$ is a coprime commutator. Since the set $J$ is infinite, we obtain that the cardinality of the set of coprime commutators of the form $\prod_{i \in I} c_{i}$ is $2^{\aleph_{0}}$. This is a contradiction and so the lemma follows.

We are now ready for the proof of Theorem 1.1 .

Proof of Theorem 1.1. Let $G$ be a profinite group. Clearly, if $G$ is finite-by-pronilpotent, then the cardinality of the set of coprime commutators in $G$ is finite.

We need to show that if $G$ is a profinite group with less than $2^{\aleph_{0}}$ coprime commutators, then $G$ is finite-by-pronilpotent. We can assume that $G$ is infinite.

Let us first show that the Fitting subgroup $F=F(G)$ of $G$ is nontrivial. Assume for a contradiction that $F=1$. If $x$ is any nontrivial coprime commutator, then by Lemma 2.1 the conjugacy class $x^{G}$ is finite. Hence $\left|G: C_{G}(x)\right|$ is finite and the same holds for any of the (finitely many) conjugates of $x$ in $G$. Therefore, $\left|G: C_{G}\left(\left\langle x^{G}\right\rangle\right)\right|$ is finite and so $\left\langle x^{G}\right\rangle$ is central-by-finite. Taking into account that $F=1$ we deduce that $\left\langle x^{G}\right\rangle$ is finite for any coprime commutator $x$. Thus, $G$ possesses minimal normal subgroups. Let $N$ be the product of all minimal normal subgroups of $G$. If $N$ is finite, then there exists an open normal subgroup $K$ of $G$ such that $K \cap N=1$. In particular, $K$ has no nontrivial minimal normal subgroups. Note that $K$ is not pronilpotent, since $F=1$. So $K$ contains a nontrivial coprime commutator and, by the above argument, its Fitting subgroup is nontrivial. This contradicts the assumption that $F=1$ and therefore $N$ is infinite.

Thus $N$ is the Cartesian product of infinitely many nonabelian finite simple groups $S_{i}$, with $i$ ranging in a set of indices $J$. Note that in each $S_{i}$ there exists a nontrivial coprime commutator $c_{i}$. Namely, take a nontrivial central element $x_{i}$ in one Sylow $p$-subgroup of $S_{i}$, for some prime $p$; then $x_{i}$ cannot commute with all Sylow $q$-subgroups of $S_{i}$, for all $q \neq p$, because otherwise $x_{i}$ would be central in $S_{i}$. For any subset $I \subseteq J$ the product $\prod_{i \in I} c_{i}$ is a coprime commutator. Since the set $J$ is infinite, we obtain that the cardinality of the set of coprime commutators of the form $\prod_{i \in I} c_{i}$ is at least $2^{\aleph_{0}}$. This is a contradiction and so we conclude that $F \neq 1$.

Observe that $F$ actually must be infinite. Indeed, if $F$ is finite, then there exists a normal subgroup $H$ of finite index such that $H \cap F=1$. On the other hand, by the above argument, the Fitting subgroup of $H$ is nontrivial, a contradiction. Thus $F$ is infinite. 
By Lemma 3.4, $\gamma_{\infty}\left(F_{2}(G)\right)$ is finite. Let $R$ be an open normal subgroup of $F_{2}(G)$ intersecting $\gamma_{\infty}\left(F_{2}(G)\right)$ trivially. As $R$ is pronilpotent, it follows that $F$ has finite index in $F_{2}(G)$. Since $F_{2}(G) / F=F(G / F)$, we deduce that $G / F$ has finite Fitting subgroup. Since we have proved above that an infinite profinite group with less than $2^{\aleph_{0}}$ coprime commutators has infinite Fitting subgroup, we conclude that $G / F$ is finite.

Now we argue by induction on the index of $F$ in $G$, the case where $G=F$ being trivial. If there exists a proper normal subgroup $N$ of $G$ properly containing $F$, then $|N: F(N)|<|G: F|$. By induction, $\gamma_{\infty}(N)$ is finite. We use the bar notation in the quotient group $\bar{G}=$ $G / \gamma_{\infty}(N)$. As $|\bar{G}: F(\bar{G})|<|G: F|$, by induction we deduce that $\gamma_{\infty}(\bar{G})$ is finite, hence $\gamma_{\infty}(G)$ is finite as well.

If $G / F$ is abelian, then $G$ is metapronilpotent and in view of Lemma 3.4 the claim is proved.

So we are left with the case where the quotient $G / F$ is nonabelian simple. Let $p$ be a prime divisor of $G / F$. Choose a $p$-element $g \in$ $G \backslash F$. Since $F\langle g\rangle$ is metapronilpotent, by Lemma 3.4 we conclude that $\gamma_{\infty}(F\langle g\rangle)$ is finite. As $\gamma_{\infty}(F\langle g\rangle)$ is normal in $F$, the normalizer of $\gamma_{\infty}(F\langle g\rangle)$ in $G$ has finite index in $G$. Thus $\left\langle\gamma_{\infty}(F\langle g\rangle)\right\rangle^{G}$ is the product of finitely many conjugates of $\gamma_{\infty}(F\langle g\rangle)$, which are all normal in $F$. Therefore, $\left\langle\gamma_{\infty}(F\langle g\rangle)\right\rangle^{G}$ is finite. Passing to the quotient over $\left\langle\gamma_{\infty}(F\langle g\rangle)\right\rangle^{G}$, we can assume that $F\langle g\rangle$ is pronilpotent. Let $\left\{t_{1}, \ldots, t_{s}\right\}$ be a transversal of $F$ in $G$ and note that each $F\left\langle g^{t_{i}}\right\rangle$ is pronilpotent. So $W=O_{p^{\prime}}(F)$ centralizes each $g^{t_{i}}$. Moreover $W$ centralizes the $p$-Sylow subgroup $P$ of $F$.

Let $G_{1}=\left\langle g^{t_{1}}, \ldots, g^{t_{s}}\right\rangle$ and note that $G=F G_{1}=W P G_{1}$. As $\left[W, P G_{1}\right]=1$, it follows that $P G_{1}$ is normal in $G$.

Note that if $\gamma_{\infty}\left(P G_{1}\right)=1$, then $G$ is pronilpotent, being a product of two normal pronilpotent subgroups. Hence $\gamma_{\infty}(G)=\gamma_{\infty}\left(P G_{1}\right)$ and, since $F \cap P G_{1}=F\left(P G_{1}\right)$, we can assume without loss of generality that $G=P G_{1}$. We still have that $G / F$ is a nonabelian simple group and now $O_{p^{\prime}}(F)$ is central in $G$. Since $G / F$ is a nonabelian simple group, we can choose a prime $q \neq p$ and a $q$-element $h$ in $G \backslash F$ and argue as above. Thus, given a transversal $\left\{r_{1}, \ldots, r_{k}\right\}$ of $F$ in $G$, we can assume that each $F\left\langle h^{r_{i}}\right\rangle$ is pronilpotent. Let $G_{2}=\left\langle h^{r_{1}}, \ldots, h^{r_{s}}\right\rangle$ and note that $G=F G_{2}$. Now we get that the Hall $q^{\prime}$-subgroup $O_{q^{\prime}}(F)$ of $F$ centralizes each $h^{r_{i}}$, hence $\left[O_{q^{\prime}}(F), G_{2}\right]=1$. Moreover $O_{q^{\prime}}(F)$ centralizes the $q$-Sylow subgroup $Q$ of $F$, hence

$$
\left[O_{q^{\prime}}(F), Q G_{2}\right]=1 \text {. }
$$

In particular $Q G_{2}$ is normal in $G=F G_{2}=O_{q^{\prime}}(F) Q G_{2}$ and thus $\gamma_{\infty}(G)=\gamma_{\infty}\left(Q G_{2}\right)$. Moreover $F\left(Q G_{2}\right)=F \cap Q G_{2}$. Note that both 
the $p^{\prime}$-Hall subgroup and the $q^{\prime}$-Hall subgroup of $F\left(Q G_{2}\right)$ are central in $Q G_{2}$, hence $F\left(Q G_{2}\right)$ is central as well. Since $F\left(Q G_{2}\right)$ has finite index in $Q G_{2}$, it follows from the theorem of Schur that $\gamma_{\infty}\left(Q G_{2}\right)=\gamma_{\infty}(G)$ is finite, as claimed.

\section{Anti-coprime commutators}

In this section we prove that a profinite group with less than $2^{\aleph_{0}}$ anti-coprime commutators has finite derived subgroup.

In 3 . the strong conciseness of several words was established. Among these are the simple commutator $[x, y]$ and the 2-Engel word $[x, y, y]=$ $[[x, y], y]$. Thus, we have

Proposition 4.1. Let $w$ be either the word $[x, y]$ or the 2-Engel word $[x, y, y]$ and let $G$ be a profinite group. Then, the verbal subgroup $w(G)$ is finite if and only if $w$ takes less than $2^{\aleph_{0}}$ values in $G$.

Proof of Theorem 1.2. As observed in the introduction, it is sufficient to show that the derived subgroup $G^{\prime}$ of a profinite group $G$ is finite whenever $G$ has less than $2^{\aleph_{0}}$ anti-coprime commutators. So, assume that $G$ is a group with this property.

Observe that, for all $g, h \in G$, the element $[g, h, h]$ is an anti-coprime commutator, since

$$
[[g, h], h]=\left[\left(h^{-1}\right)^{g} h, h\right]=\left[\left(h^{-1}\right)^{g}, h\right]^{h} .
$$

Therefore, since $G$ has less than $2^{\aleph_{0}}$ anti-coprime commutators, there are less than $2^{\aleph_{0}}$ values of the 2-Engel word in $G$. It follows from Proposition 4.1 that the verbal subgroup $N$ generated by the values of the 2-Engel word is finite. Passing to the quotient $G / N$, we can assume that $[g, h, h]=1$ for every $g, h \in G$, so $G$ is a 2-Engel group. Since finite Engel groups are nilpotent (see [12, 12.3.4]), the group $G$ is pronilpotent.

As $G$ is a direct product of its Sylow subgroups, every simple commutator $[g, h]$ is an anti-coprime commutator. Indeed, we can always write $h=h_{1} h_{2}$ and $g=g_{1} g_{2}$, where $\pi\left(g_{1}\right)=\pi\left(h_{1}\right)$ while $\left(\left|h_{2}\right|,|g|\right)=\left(|h|,\left|g_{2}\right|\right)=1$. Then simply observe that $[g, h]=\left[g_{1}, h_{1}\right]$ is an anti-coprime commutator.

So $G$ has less than $2^{\aleph_{0}}$ simple commutators $[g, h]$. Using Proposition 4.1 we conclude that $G^{\prime}$ is finite, as required.

\section{Acknowledgments}

The authors thank the referee for his suggestions. The first and second author are members of INDAM and were partially supported by BIRD185350/18; the third author was partially supported by FAPDF 
and CNPq-Brazil.

\section{References}

[1] C. Acciarri, P. Shumyatsky, A. Thillaisundaram, Conciseness of coprime commutators in finite groups, Bull. Aust. Math. Soc. 89 (2014), 252-258.

[2] Aschbacher, M., Finite group theory. Second edition. Cambridge Studies in Advanced Mathematics, 10. Cambridge University Press, Cambridge, 2000.

[3] E. Detomi, B. Klopsch, P. Shumyatsky, Strong conciseness in profinite groups, to appear in J. London Math. Soc. (2), arXiv:1907.01344.

[4] E. Detomi, M. Morigi, P. Shumyatsky, Words of Engel type are concise in residually finite groups. Bull. Math. Sci. 9 (2019), 1950012, 19 pp.

[5] E. Detomi, M. Morigi, P. Shumyatsky, Words of Engel type are concise in residually finite groups. Part II, to appear in Groups Geom. Dyn., arXiv:1905.07784

[6] I. M. Isaacs, Finite Group Theory, Graduate Studies in Mathematics, 92. American Mathematical Society, Providence, RI, 2008.

[7] J. L. Kelley, General Topology, Van Nostrand, Toronto - New York - London, 1955.

[8] E. I. Khukhro, P. Shumyatsky, Almost Engel finite and profinite groups, Internat. J. Algebra Comput. 26 (2016), 973-983.

[9] L. Lévai, L. Pyber, Profinite groups with many commuting pairs or involutions, Arch. Math. (Basel) 75 (2000), 1-7.

[10] C. D. Reid, Local Sylow theory of totally disconnected, locally compact groups, J. Group Theory 16 (2013), 535-555.

[11] L. Ribes, P. Zalesskii, Profinite groups, A Series of Modern Surveys in Mathematics, 40. Springer-Verlag, Berlin, 2000.

[12] D. J. S. Robinson, A course in the theory of groups, Second edition. Graduate Texts in Mathematics, 80. Springer-Verlag, New York, 1996.

[13] D. J. S. Robinson, Finiteness conditions and generalized soluble groups. Part 1. Springer-Verlag, New York-Berlin, 1972.

[14] J. S. Wilson, Profinite groups, Clarendon Press, Oxford, 1998.

Dipartimento di Ingegneria dell'Informazione - DEI, Università di

Padova, Via G. Gradenigo 6/B, 35121 Padova, Italy

Email address: eloisa.detomi@unipd.it

Dipartimento di Matematica, Università di Bologna, Piazza di Porta

San Donato 5, 40126 Bologna, Italy

Email address: marta.morigi@unibo.it

Department of Mathematics, University of Brasilia, Brasilia-DF, 70910-900 BRAZIL

Email address: pave12040@gmail.com 